
CA1 is required for retrieving gist memories over time and CA3 for memory precision

Erika Atucha ${ }^{1}$, Shih-Pi Ku ${ }^{1}$, Michael T. Lippert ${ }^{1}$, Frank W. Ohl ${ }^{1}$ Magdalena M. Sauvage ${ }^{1,2,3}$

${ }^{1}$ Leibniz-Institute for Neurobiology, Functional Architecture of Memory Dept., Magdeburg, Germany,

2Otto von Guericke University, Medical Faculty, Functional Neuroplasticity Dept., Magdeburg Germany erikaatutxa@gmail.com and magdalena.sauvage@gmail.com

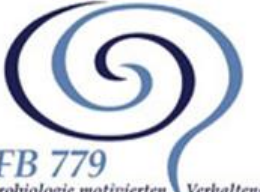

\section{AIM}

We aim to investigate the contribution of CA1 and $\mathrm{CA} 3$ to the nature of recent and remote memories.

\section{METHOD}

Fear conditioning task to investigate memory precision in combination with CA1 or CA3 optogenetic inhibition (ArchT) and immediate early gene Arc imaging.

Conditioning
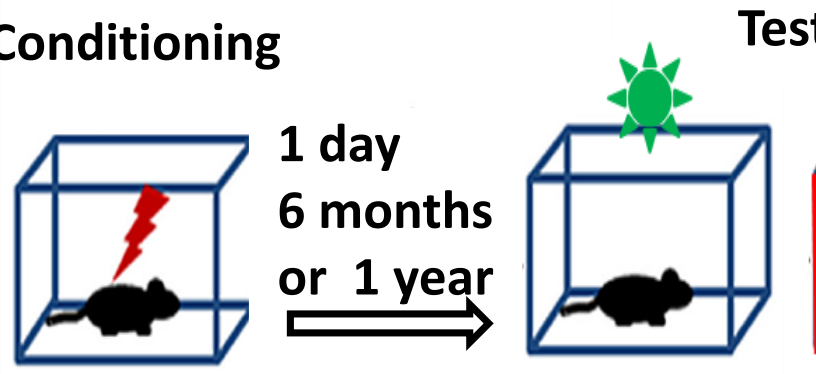

Conditioning context

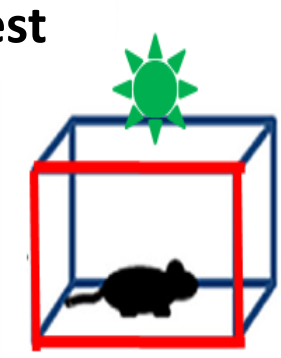

Safe

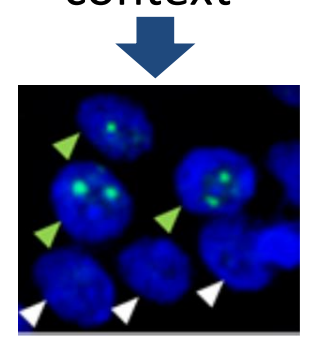

Arc imaging only after reexposure to the conditioning context

\title{
MEMORY PERFORMANCE
}

CA3 is required for the precision of recent memory

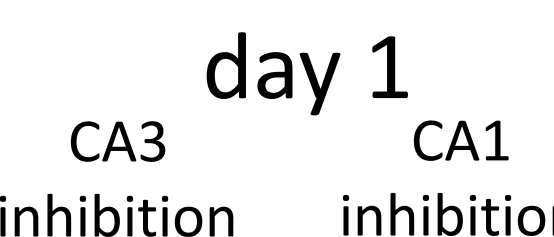

CA1 is crucial for remote gist memory

6 months

CA3 CA1

inhibition inhibition

1 year

CA3 CA1

inhibition inhibition

$\square$ Saline $\square$ No light control $\square$ Light (ArchT inhibition)
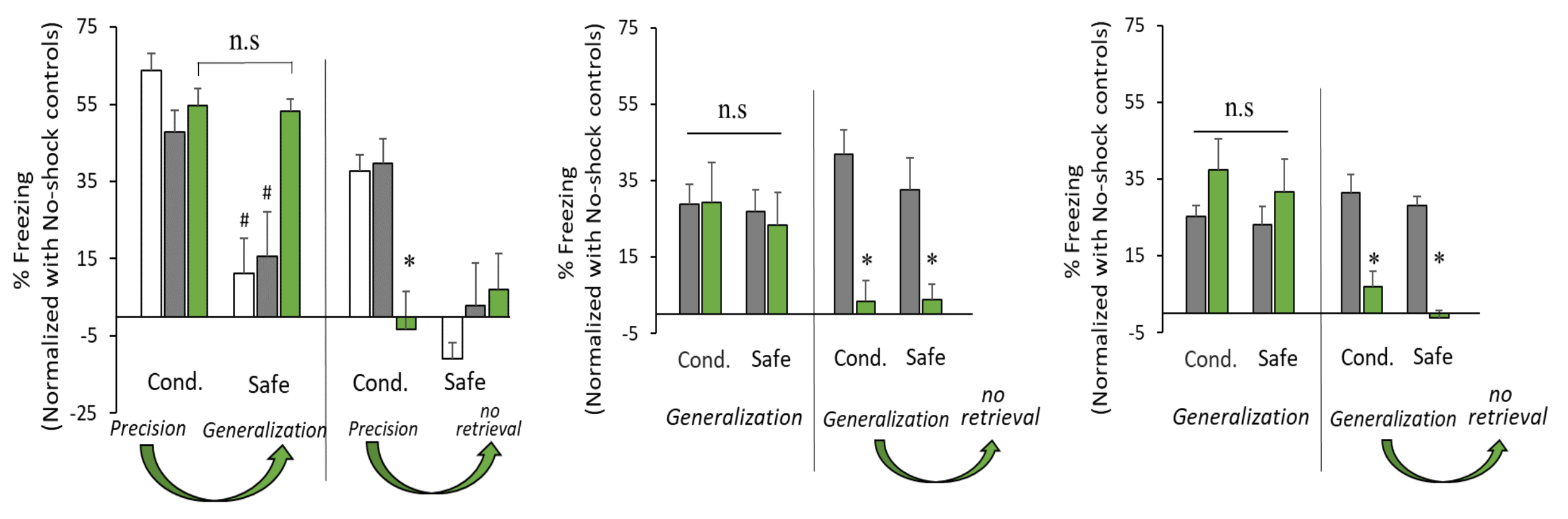

\section{ARC IMAGING RESULTS AND CONCLUSIONS}

CA3 inhibition has a local effect only disrupting Arc activity in CA3 during recent memory retrieval:

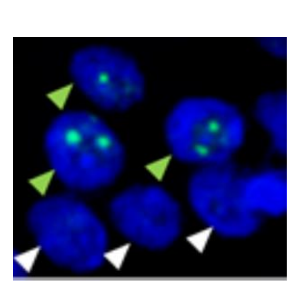

Retrieval of precise memory requires CA3.

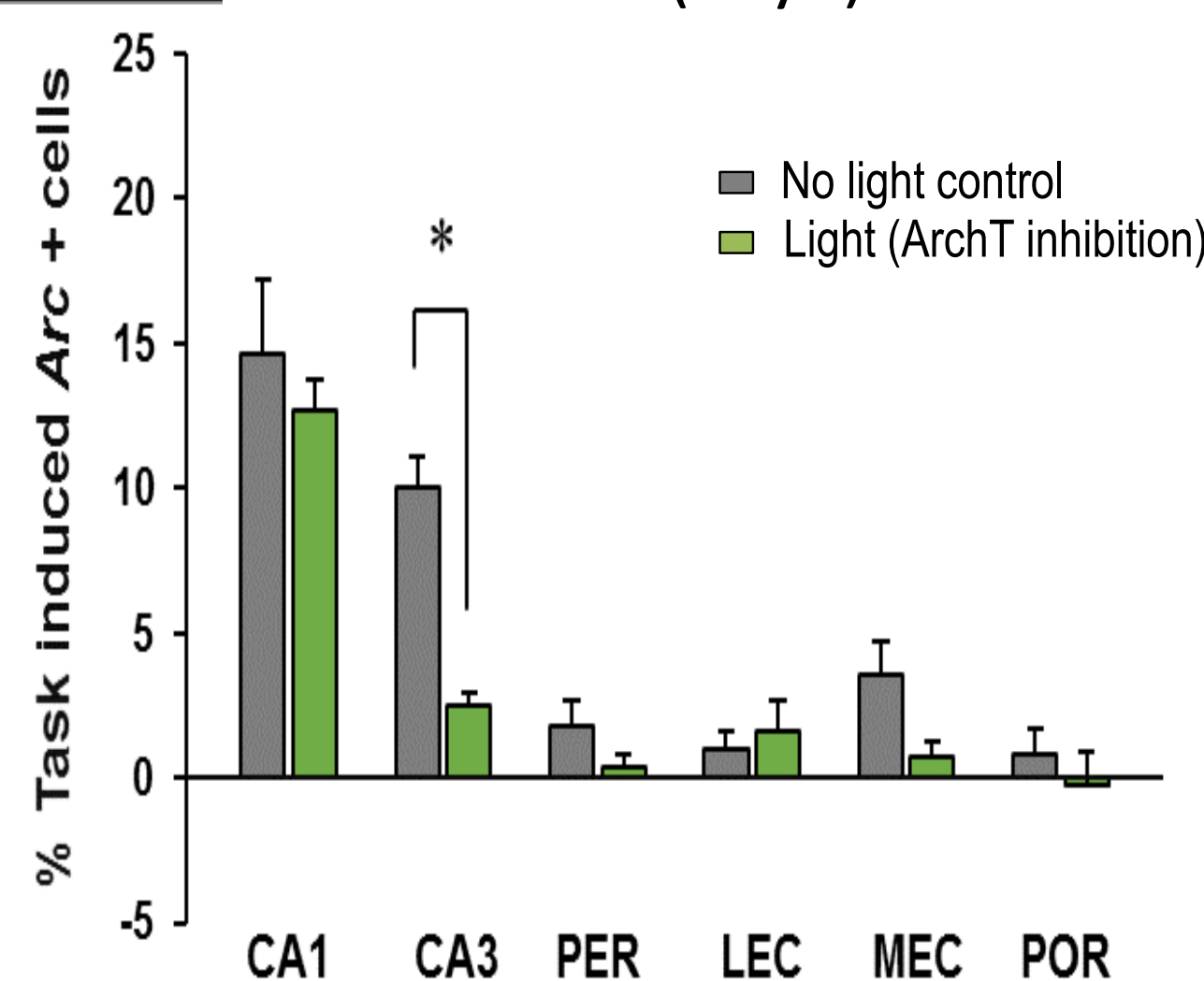

Inhibiting CA3 has no effect on remote memory retrieval

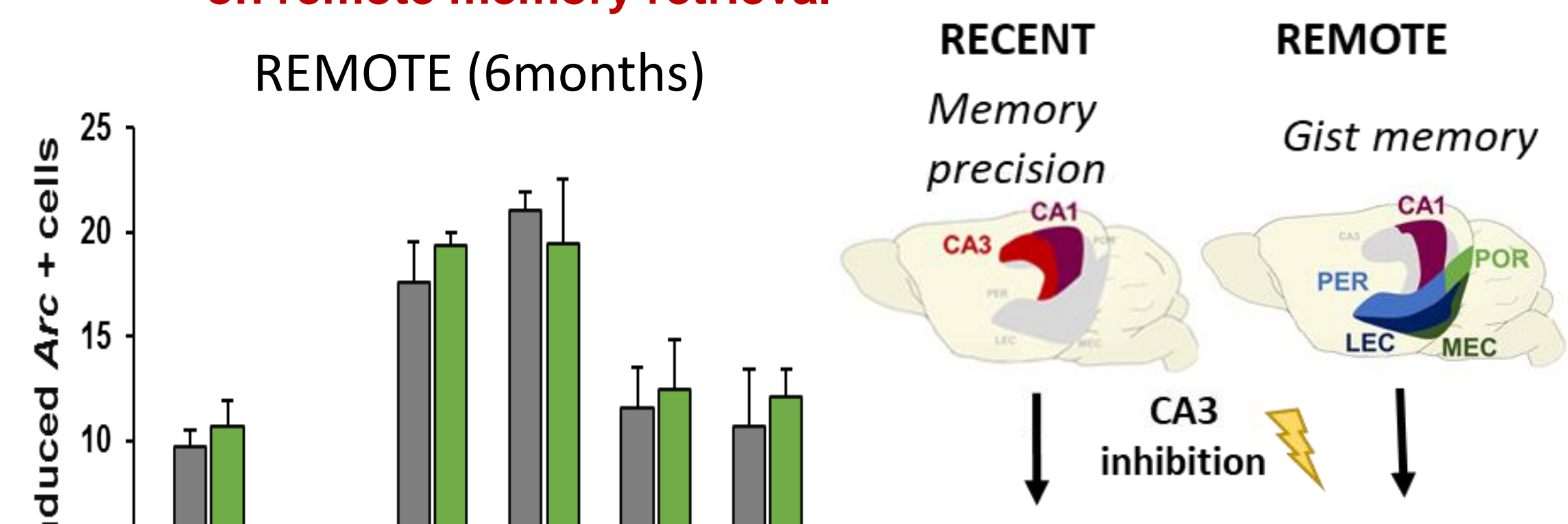

Gist memory

Gist memory

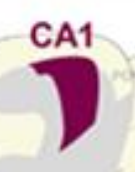

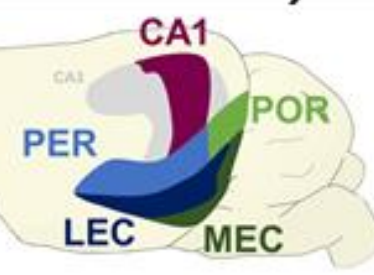

CA1 inhibition has a global effect disrupting Arc activity in the engaged regions during memory retrieval:

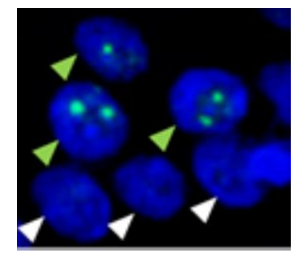

$\stackrel{(n)}{=}$

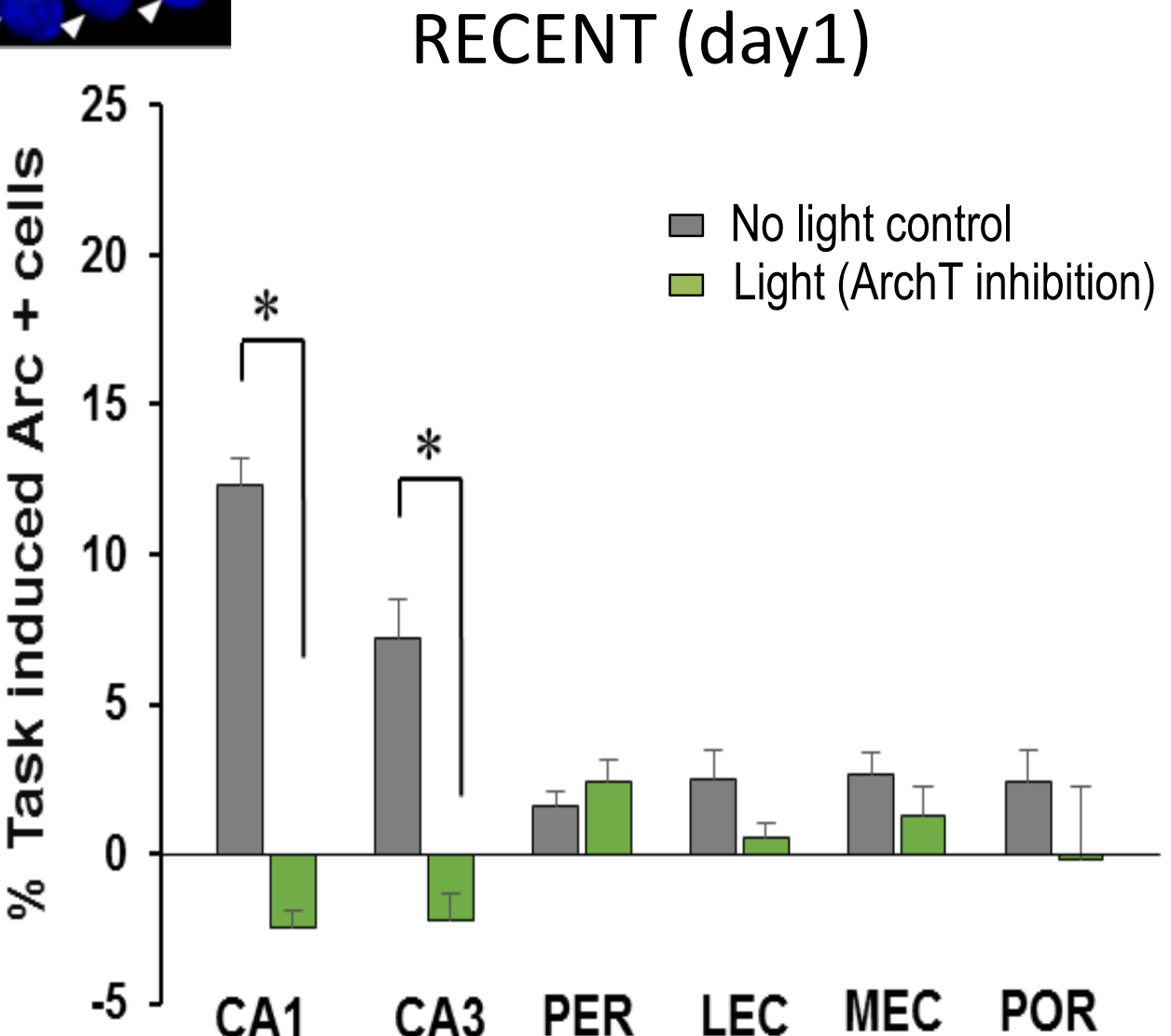

RECENT (day1)

CA1 is required for retrieving recent and remote gist memories.

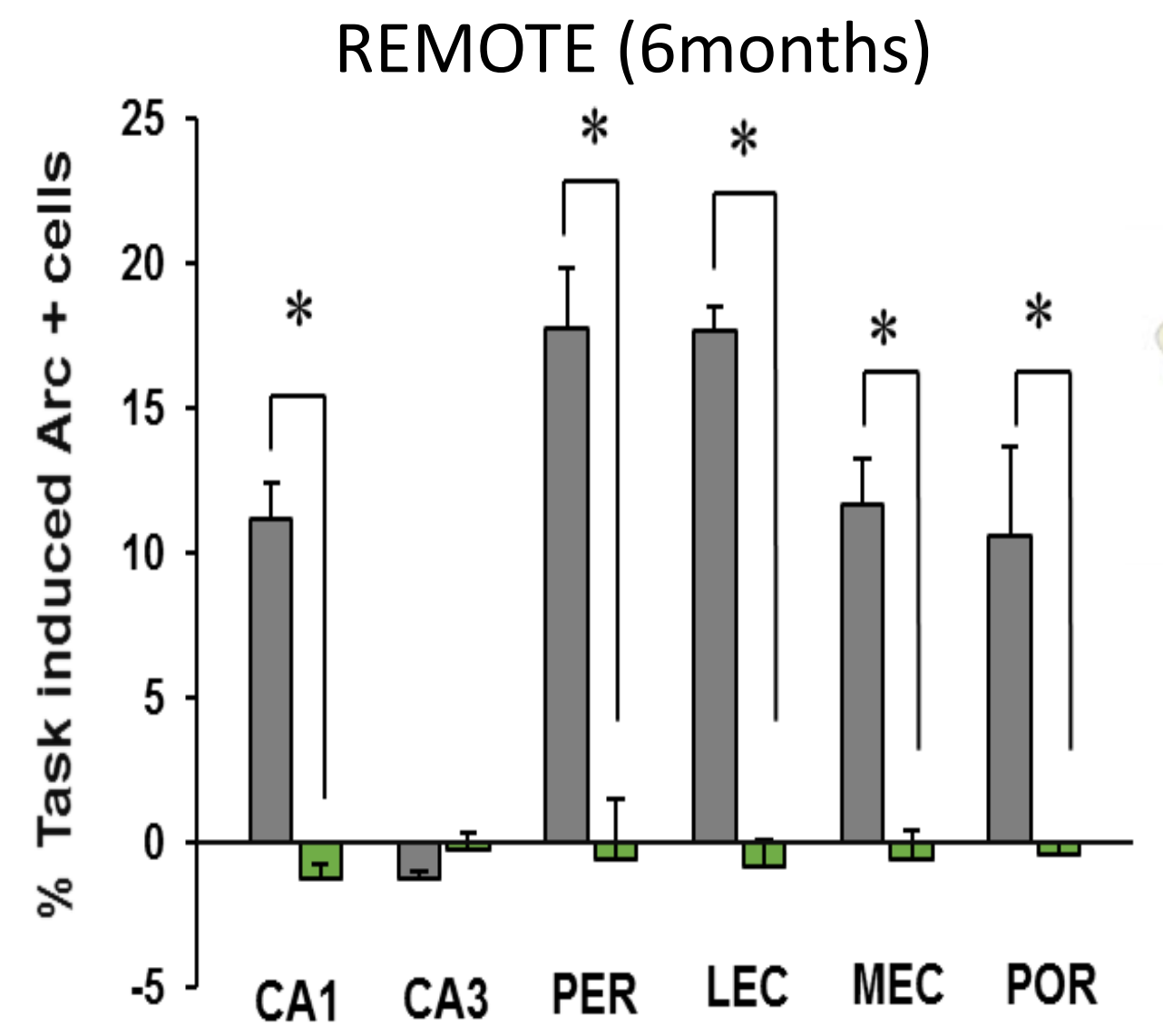

RECENT

REMOTE

Memory

precision

CA3

Gist memory

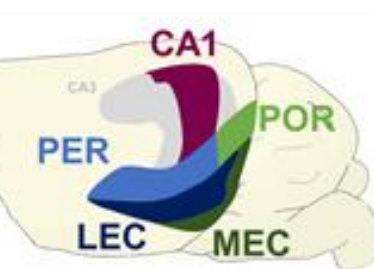

$\downarrow$ inhibition

No retrieval

No retrieval 